Canad. J. Math. Vol. 55 (2), 2003 p. 247

\title{
Differential Structure of Orbit Spaces: Erratum
}

\author{
Richard Cushman and Jędrzej Śniatycki
}

Abstract. This note signals an error in the above paper by giving a counter-example.

Theorem 5.4 and consequently Corollary 5.5 are incorrect as can be seen from the following example.

Let $G=\mathrm{SO}(2)$ act on $P=\mathbb{R}^{2}$ by rotations around the origin. The $G$-orbit space $\bar{P}$ is diffeomorphic to the half-line $[0, \infty)$, which is the union of two strata $\{0\}$ and $(0, \infty)$. The natural differential structure $C^{\infty}(\bar{P})$ of $\bar{P}$ can be described as the collection of functions on $[0, \infty) \subset \mathbb{R}$, which are restrictions of smooth functions on $\mathbb{R}$. The derivation $\frac{\partial}{\partial x}$ on $C^{\infty}(\mathbb{R})$ restricts to a derivation $X$ on $C^{\infty}(\bar{P})$. Hence, $X(0) \in T_{0} \bar{P}$ and $X(0) \neq 0$. But $T_{0}\{0\}=0$.

This error does not affect any other result in the paper.

email:cushman@math.uu.nl

Received by the editors August 19, 2002.

AMS subject classification: Primary: 37J15; secondary: 58A40, 58D19, 70H33.

(c) Canadian Mathematical Society 2003. 